\title{
BOOP presenting with haemoptysis and multiple cavitary nodules
}

\author{
M. Froudarakis*, D. Bouros*, R. Loire**, K. Valasiadou+, D. Tsiftsis ${ }^{+}$, N.M. Siafakas*
}

BOOP presenting with haemoptysis and multiple cavitary nodules. M. Froudarakis, $D$. Bouros, R. Loire, K. Valasiadou, D. Tsiftsis, N.M. Siafakas. (CERS Journals Ltd 1995 ABSTRACT: A 47 year old woman developed idiopathic bronchiolitis obliterans organizing pneumonia (BOOP) presenting with haemoptysis and diffuse multiple cavitary nodules.

The disease was histologically confirmed by open lung biopsy after other entities had been excluded. The patient responded to a course of corticosteroids. ules.

Eur Respir J., 1995, 8, 1972-1974.

Bronchiolitis obliterans organizing pneumonia (BOOP) is a pulmonary disorder characterized by plugs of granulation tissue in bronchioles, alveolar ducts and alveoli. It may be associated with viral infection, toxic fume inhalation, connective tissue disease, drug administration, bone marrow and heart-lung transplantation [1]. In most cases, the cause remains unknown. Most patients improve over a period of months, with an overall mortality of 5\% [2]. Haemoptysis as a presenting symptom associated with a nodular pattern is rare [1]. To our knowledge, this is the only second report of BOOP presenting with haemoptysis and multiple diffuse cavitary nodules [3].

\section{Case report}

A 47 year old female, nonsmoker was admitted to the hospital due to cough and haemoptysis. The patient had been well until 2 months before her hospitalization, when she developed severe dry cough. This complaint progressively increased with the appearance of bloody sputum a few days before admission. She denied dyspnoea, fever, weight loss, or other systemic signs. History did not reveal any exposure to fume or other inhaled irritants. On admission, clinical examination revealed fine end-inspiratory crackles in both lower pulmonary fields. The patient had an excellent performance status, and no dyspnoea or tachypnoea were noted (respiration rate 16 breaths $\left.\cdot \mathrm{min}^{-1}\right)$.

Laboratory data on admission showed: erythrocyte sedimentation rate (ERS) $36 \mathrm{~mm} \cdot \mathrm{h}^{-1}$, white blood cells (WBC) $6 \times 10^{9}$ cells $\cdot \mathrm{L}^{-1}$ (neutrophils $55 \%$, lymphocytes $35 \%$, monocytes $5 \%$, eosinophils $3 \%$ and basophils $2 \%$ ).
Depts of *Thoracic Medicine and +Surgical Oncology, Medical School, University of Crete, Greece. ${ }^{* *}$ Dept of Pathology, Louis Pradel Hospital, Claude Bernard University, Lyon, France.

Correspondence: M. Froudarakis, Dept of Thoracic Medicine, University Hospital Heraklion, Crete, 71110 Greece

Keywords: Bronchiolitis obliterans organizing pneumonia, cavitary nodules, cavitation, haemoptysis

Received: October 281994

Accepted after revision July 201995
Creatinine was $85 \mathrm{mmol} \cdot \mathrm{mL}^{-1}$, and blood urea nitrogen (BUN) was $0.33 \mathrm{mg} \cdot \mathrm{mL}^{-1}$. Chest radiography showed multiple round nodules on both lungs (fig. 1). High resolution computed tomography (HRCT) of the chest showed multiple excavating nodules of 10-20 mm diameter, predominantly in the middle and lower parts of the lungs (fig. 2).

Pulmonary function tests indicated a minor restrictive defect with a normal CO transfer test. Arterial blood gases demonstrated a moderate hypoxaemia (arterial oxygen tension $\left.\left(\mathrm{Pa}, \mathrm{O}_{2}\right) 11.3 \mathrm{kPa}(85 \mathrm{mmHg})\right)$, which increased on exercise $\left(\mathrm{Pa}_{\mathrm{a}} \mathrm{O}_{2} \mathrm{ex} 10.1 \mathrm{kPa}(76 \mathrm{mmHg})\right)$. Fibreoptic bronchoscopy revealed a normal bronchial mucosa. Bronchial washing and biopsy were normal. Bronchoalveolar lavage showed an increased number of

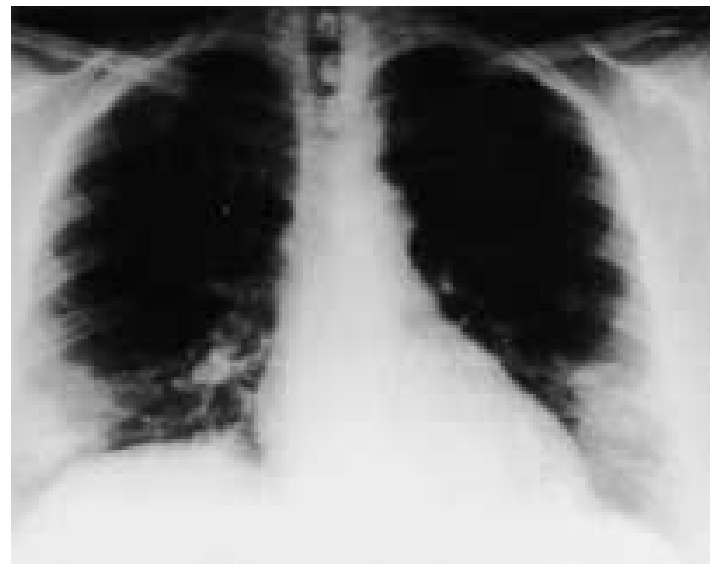

Fig. 1. - Plain chest roentgenogram showing multiple round nodules on both lungs. 
a)

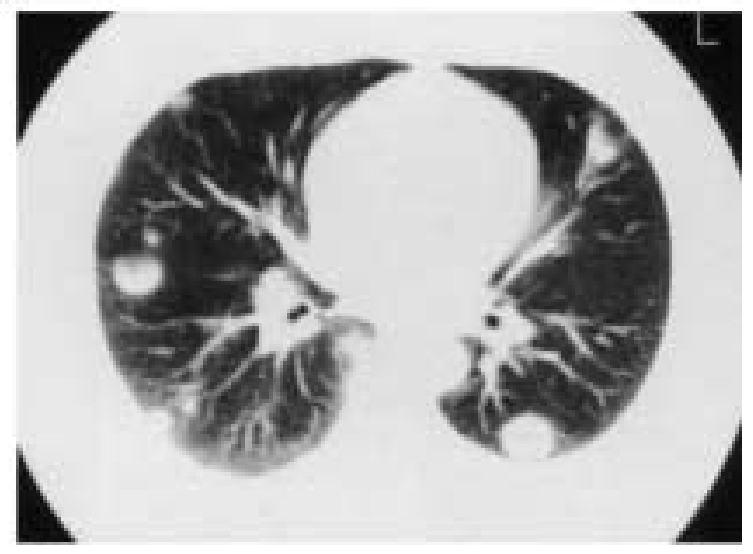

b)

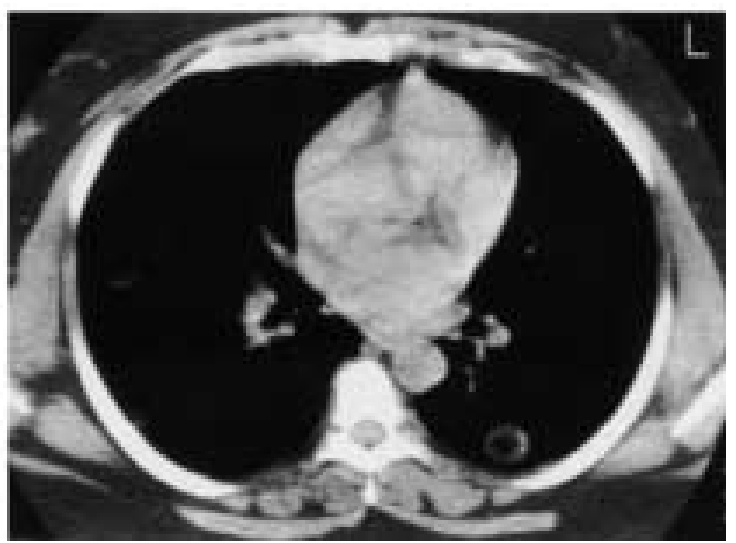

Fig. 2. - High-resolution computed tomography of the chest showing multiple cavitary nodules. a) lung window; b) bone window.

recovered cells, with macrophages $75 \%$ and lymphocytes $25 \%$.

The upper respiratory tract and the eyes, checked by clinical examination and computed tomography (CT), were normal. An abdominal and pelvic CT did not reveal any abnormality, nor did mastography. Levels of tumour markers (carbohydrate antigen (CA) 15-3, carcinoembryonic antigen (CEA), CA 125, CA 19-9, alpha-fetoprotein (AFP), thyroglobulin, calcitonin) were within the normal range. Cytology of sputum was normal. Microbiology specimens (blood and sputum cultures and serology for Mycoplasma, Rickettsiae, Chlamydiae, Legionella, viruses, mycosis) were negative. Renal function was normal, and no proteinuria or haematuria were noted. Serum levels of immunogloblin E (IgE), rheumatoid factor, anti-deoxyribonucleic acid (DNA) antibodies, circulating immune complexes and antineutrophilic antibodies were repeatedly negative.

An open lung biopsy was performed, in the left lower lobe, resecting an entire nodule. Histology of the nodule showed the presence of polyps of connective tissue within alveolar ducts and bronchioles, associated with inflammatory infiltration of the bronchioles and the surrounding interstitium. Necrosis was not observed in the resected specimen. The findings were indicative of BOOP

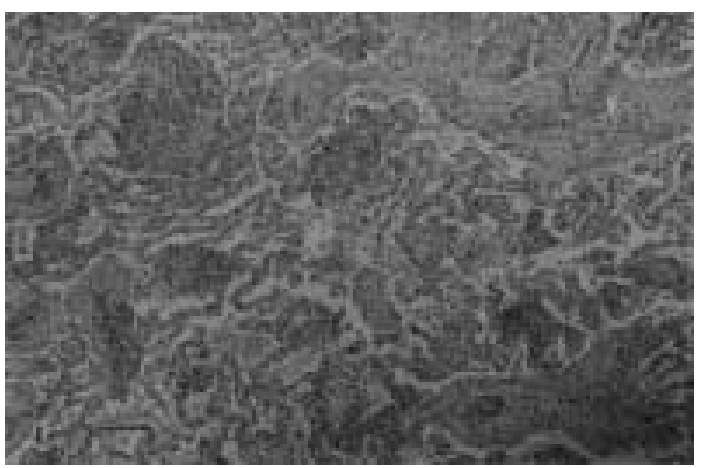

Fig. 3. - Pathological specimen of the open lung biopsy, showing the pattern of distribution of pulmonary and bronchiolar lesions of bronchiolitis obliterans organizing pneumonia (BOOP). (Internal scale bar $=100 \mu \mathrm{m})$. Original magnification $\times 25$ ).

(fig. 3). The patient was prescribed prednisone $\left(1 \mathrm{mg} \cdot \mathrm{kg}^{-1}\right)$. One month later, the cough disappeared, and the chest roentgenogram and HRCT became normal.

Prednisone was progressively decreased 6 months after initiation and withdrawn one year after initiation. Two years after the initial event and 10 months after the complete cessation of prednisone, the patient remained clinically asymptomatic and the radiographic control was normal.

\section{Discussion}

BOOP is defined pathologically by the presence within the lumen of distal air spaces (bronchioles, alveolar ducts and alveoli) of granulation tissue composed mainly of fibroblasts and connective matrix [4]. The illness occurs in men and women aged 20-70 yrs and is not related to smoking [2].

Common clinical features of BOOP are cough and dyspnoea in about $75 \%$ of cases [5, 6]. A flu-like illness is also common $[5,6]$. Wheezing is the presenting symptoms in less than 5\% of cases [5, 6]. EPLER et al. [7] reported just one case of haemoptysis in their series of 67 patients $(1.5 \%)$. Fine end-inspiratory crackles are heard in about $75 \%$ of patients $[5,6]$. A febrile illness with inflammatory syndrome (increased sedimentation rate) is also common according to several studies $[6,7]$. The duration of illness is less than 3 months in $75 \%$ of patients [6].

Multiple cavities, as observed in our case, are very rare. Typical radiological findings are bilateral patchy infiltrates in $80 \%$ of cases, bilateral linear in $12 \%$, whilst round nodular opacities are less frequent (7\%) [7]. Cavities and effusions are uncommon [7]. CORDIER et al. [8] noted that patients with idiopathic BOOP and diffuse small nodules had a chronic disease and a worse prognosis, whilst patients with patchy infiltrates and inflammatory syndrome responded to corticosteroid therapy of a sufficient amount and duration. MüLLER and co-workers $[9,10]$ did not observe a case of cavitary nodules in their series. On CT of the chest, their patients presented air space consolidation, small nodular opacities, 
bronchial wall thickening and dilatation $[9,10]$. However, in our patient the CT scan of the lung revealed cavitary lesions which were not shown on the plain chest radiograph. CT scan is more sensitive than plain chest radiography, for revealing parenchymal lesions [11].

Allegre-Martin et al. [3] described three cases of idiopathic BOOP, one of which had a cavitary nodular disease associated with haemoptysis, but no further information was given. It was reported that bleomycin, given for sarcoma or germ cell carcinoma, could be the cause of BOOP simulating metastatic disease [12-14]. A metastatic carcinoma was excluded by the pathological specimen of the open lung biopsy.

Wegener's disease is a differential diagnosis of BOOP [15] and the cavity lesions of our patient were reminiscent of this disease, but the pathological examination of the lung biopsy in our patient did not reveal any necrotizing granulomatous lesions or vasculitic features. There were also no systemic manifestations of the disease.

In our patient, an initial infectious process with slowly resolving lesions was possible, but the absence of acute febrile symptoms, the progressive worsening of her complaints, as well as the repeatedly negative microbiological tests, make this possibility less likely. Also, on the extracted nodule the pathological examination did not reveal any necrosis or marked infiltration of polymorphonuclear lymphocytes, and the microbiological examination remained sterile $[15,16]$.

Although the clinical and radiological presentation of BOOP has shown a distinct pattern in the different series $[7,8]$, our case indicates that an unusual presentation is possible. The clinician aware of this entity should not miss the diagnosis in such cases, and should proceed to open lung biopsy for diagnostic confirmation. BOOP presenting with haemoptysis and multiple cavities may mimic Wegener's disease or metastatic lung disease. Differential diagnosis is important since BOOP usually shows a good response to cortcosteroids, as was seen in our case.

\section{References}

1. Epler GR. Bronchiolitis obliterans organizing pneumonia: definition and clinical features. Chest 1992; 102 (Suppl 1): 2s-6s.
2. Epler GR, Colby TV. The spectrum of bronchiolitis obliterans. Chest 1983; 83: 161-162.

3. Allegre-Martin J, Fernandez de Sevilla T, Garcia F, et al. Three cases of idiopathic bronchiolitis obliterans with organizing pneumonia. Eur Respir J 1991; 4: 902904.

4. Cordier JF, Peyrol S, Loire R. Bronchiolitis obliterans organizing pneumonia as a model of inflammatory lung disease. In: Epler GR, ed. Diseases of the Bronchioles. New York, Raven Press Ltd, 1994; pp. 313345.

5. King TE Jr, Mortenson RL. Cryptogenic organizing pneumonitis: the North American experience. Chest 1992; 102 (Suppl. 1): 8s-13s.

6. Costabel U, Teschler H, Schoenfeld B, et al. BOOP in Europe. Chest 1992; 102 (Suppl. 1): 14s-20s.

7. Epler GR, Colby TV, McLoud TC, Gaensler EA. Bronchiolitis obliterans organizing pneumonia. N Engl J Med 1985; 312: 152-158.

8. Cordier JF, Loire R, Brune J. Idiopathic bronchiolitis obliterans organizing pneumonia. Chest 1989; 96: 999-1004.

9. Müller NL, Guerry-Force ML, Staples CA, et al. Differential diagnosis of bronchiolitis obliterans with organizing pneumonia: clinical, functional and radiologic findings. Radiology 1987; 162: 151-156.

10. Müller NL, Staples CA, Miller R. Bronchiolitis obliterans organizing pneumonia: CT features in 14 patients. Am J Roentgenol 1990; 154: 983-987.

11. Nishimura K, Itoh H. High-resolution computed tomographic features of bronchiolitis obliterans organizing pneumonia. Chest 1992; 102 (Suppl. 1): 26s-31s.

12. Nachman JB, Baum ES, White H, Cruissi FG. Bleomycin-induced pulmonary fibrosis mimicking recurrent metastatic disease in a patient with testicular carcinoma: case report of the CT scan appearance. Cancer 1981; 47: 236-239.

13. Cohen MB, Austin JH, Smith-Vaniz A, Lutzky J, Grimes MM. Nodular bleomycin toxicity. Am J Clin Pathol 1989; 92: 101-104.

14. Santrach PJ, Askin FB, Wells RJ, Azizkhan RG, Merten DF. Nodular form of bleomycin-related pulmonary injury in patients with osteogenic sarcoma. Cancer 1989; 64: 806-811.

15. Kaitachi M. Differential diagnosis of bronchiolitis obliterans organizing pneumonia. Chest 1992; 102 (Suppl. 1): $44 \mathrm{~s}-49 \mathrm{~s}$.

16. Colby TV. Pathologic aspects of bronchiolitis obliterans organizing pneumonia. Chest 1992; 1021 (Suppl. 1): $38 \mathrm{~s}-43 \mathrm{~s}$. 